\title{
Direct evidence for the occurrence of superconductivity in the magnetic compound $\mathrm{YFe}_{4} \mathrm{Al}_{8}$
}

\author{
V. M. Dmitriev ${ }^{1,2,3}$, L. F. Rybaltchenko ${ }^{1,2}$, P. Wyder $^{1}$, A. G. M. Jansen ${ }^{1}$, \\ N. N. Prentslau ${ }^{2}$, and W. Suski $i^{3,4}$ \\ ${ }^{1}$ Grenoble High Magnetic Field Laboratory, Max-Planck-Institut für Festkörperforschung and \\ Centre National de la Recherche Scientifique, B.P. 166, F-38042 Grenoble Cedex 9, France \\ ${ }^{2}$ B. Verkin Institute for Low Temperature Physics and Engineering of the National Academy of \\ Sciences of Ukraine, 47 Lenin Ave., Kharkiv 61103, Ukraine \\ E-mail: dmitriev@ilt.kharkov.ua \\ ${ }^{3}$ International Laboratory of High Magnetic Fields and Low Temperatures, \\ 53-421 Wroclaw, Poland
}

${ }^{4}$ W. Trzebiatowski Institute of Low Temperatures and Structure Research Polish Academy of Sciences, 50-950 Wroclaw, Poland

Received December 28, 2002

\begin{abstract}
For the first time we present the direct evidence for superconductivity in the ternary magnetic compound $\mathrm{YFe}_{4} \mathrm{Al}_{8}$ with the $\mathrm{ThMn}_{12}$ type structure found via point-contact (PC) experiments on contacts between silver needle and single-crystal $\mathrm{YFe}_{4} \mathrm{Al}_{8}$, revealing the distinct Andreev-reflection current. The spectra measured prove the existence of normal-superconducting interface and exhibit the triangular-like shape in a vicinity of zero-bias voltage, inferring the unconventional type of superconductivity. The derived dependences of the order parameter versus temperature $\Delta(T)$ and magnetic field $\Delta(H)$ are presented. $\Delta(T)$ follows BCS theory, whereas $\Delta(H)$ do not satisfy any theoretical predictions. In some cases there exists noticeable superconductivity enhancement by a weak magnetic field. The data obtained imply the very inhomogeneous distribution of superconductivity over the sample volume in spite of its single crystal structure. We assume that the reason is associated with inherent magnetic inhomogeneities of this material. The highest values for the critical temperature $T_{c}$, upper critical magnetic field $H_{c 2}$, and ratio $2 \Delta(0) / k T_{c}$ are $7.4 \mathrm{~K}, 5 \mathrm{~T}$, and 7.2 , respectively.
\end{abstract}

PACS: 74.70.Ad, 74.80.Fp

So far there have been discovered many materials where superconductivity and magnetism coexist in a wide temperature range. Among them, for example, there are ternary Chevrel phase and related systems [1], borocarbide and boronitride compounds [2], and ruthenate cuprate family [3]. In this work we give, for the first time, the direct evidence for the existence of superconductivity in the rare-earth ternary magnetic compound $\mathrm{YFe}_{4} \mathrm{Al}_{8}$ in an Andreevreflection experiment.
The family of ternary magnetic compounds $\mathrm{ReM}_{4} \mathrm{Al}_{8}$ ( $\mathrm{Re}$ is a rare earth, $\mathrm{M}$ is a transition metal) with the $\mathrm{ThMn}_{12}$ type structure is known for about two decade and their physical properties have been investigated extensively [4-6]. However, weak signs of superconductivity in some of the compounds belonging to this family were discovered only recently in the radio-frequency impedance and heat capacity experiments $[7,8]$. This is very surprising, because the compounds of this type have a 
complicated magnetic structure and their magnetic ordering is far from a completely compensated antiferromagnetic (AFM) order for which superconductivity may coexist with magnetism. For compounds with a nonmagnetic Re-element, like $\mathrm{YFe}_{4} \mathrm{Al}_{8}$, the incommensurate AFM structure consists of Fe moments in the (001) plane forming a rotating spiral structure. Besides, a migration of $\mathrm{Fe}$ atoms can create locally a noticeable excess of magnetic moments leading to the formation of a ferrimagnetic state or a spin-glass state [4-6]. These factors cause the appearance of significant noncompensated magnetic moments which in general prevent the possible occurrence of superconductivity in such systems.

For a more direct proof of superconductivity in $\mathrm{YFe}_{4} \mathrm{Al}_{8}$, we have undertaken an Andreev-reflection study using pressure-type point contacts (PC). As is well known, the Andreev reflection of the quasiparticles, passing a normal-metal/superconductor interface, leads to the lowering of the contact resistance for an applied voltage smaller than the superconducting order parameter $\Delta$ (i.e., $e V<\Delta$ ) [9]. The measurements of the PC differential resistance characteristics, $d V / d I(V)$, were performed on contacts of the needle-anvil geometry with a silver needle as the normal electrode and freshly fractured surfaces of the $\mathrm{YFe}_{4} \mathrm{Al}_{8}$ single crystal as the superconducting counterelectrode. The contact sizes varied within $10-100 \mathrm{~nm}$. The standard modulation techniques were used for the registration of $d V / d I(V)$ characteristics.

In some parts of fractured Y-compound surfaces (about 5\%), we have found evident signatures of superconductivity. In Figs. 1 and 2 we present the typical spectra measured, respectively, at different temperatures and magnetic fields which can be seen as the first direct evidence for the superconductivity in this material. As one can see in Fig. 1, the weak zero bias minimum, arised firstly at $6.8 \mathrm{~K}$ curve and growing in amplitude with the temperature lowering, is a clear sign of probing the superconducting area. Relatively small reduction of $d V / d I(V)$ near $V=0$ implies that only a small part of PC area goes to the superconducting state. Meanwhile, in some cases this reduction could achieve about 30\% that indicates for very nonuniform distribution of superconductivity over sample volume. As is seen, the triangular shape of $d V / d I(V)$ minimum observed in a vicinity of zero voltage deviates strongly from the standard shape, i.e., expected from BTK [9] theory for contacts with $s$-wave superconductors. In the latter case, the shape of this reduction should look like a double minimum characteristic, if an electron scattering at $N-S$ boundary occurs, or should have

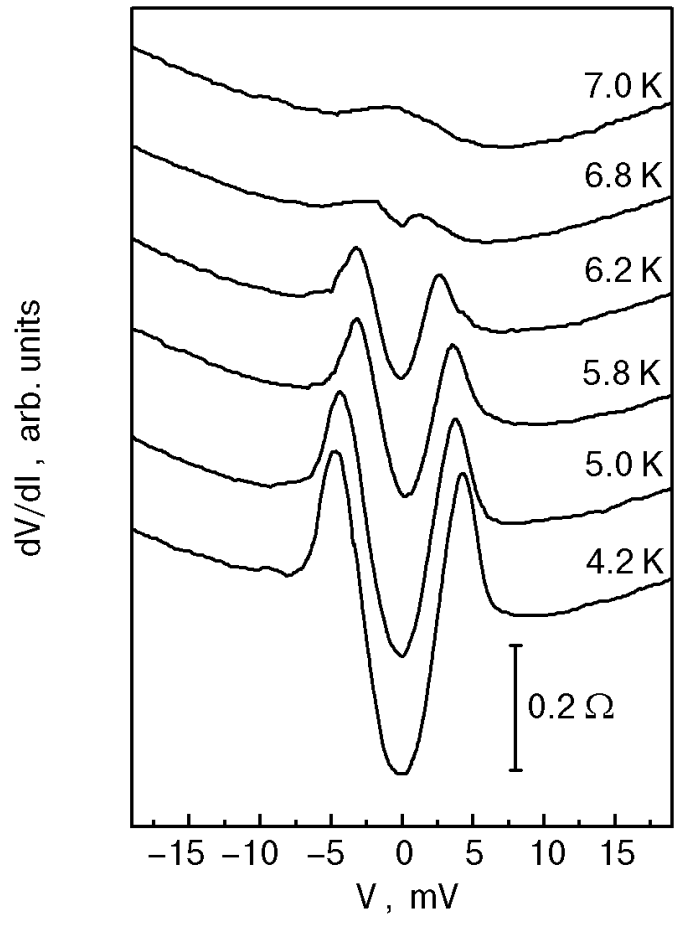

Fig. 1. $d V / d I(V)$ spectra at the different indicated temperatures for a point contact between an Ag needle and $\mathrm{YFe}_{4} \mathrm{Al}_{8}$ single crystal with normal contact resistance $R_{n}=27 \Omega$. For clarity the curves are shifted vertically. The zero-bias structure disappears at a critical temperature $T_{c}=6.84 \mathrm{~K}$.

a flat bottom when this scattering is absent. The triangular shape of spectrum may be caused by a magnetic pair-breaking scattering intrinsic in given compound or reflect the unconventional Cooperpairing, like in $\mathrm{UPt}_{3}$ [10].

All the spectra are also characterized by the prominent horn structure which is often observed in low ohmic contacts. The reason of their appearance may be associated with the fast decrease of the Andreev-reflection current near $e V=\Delta$ and chargeimbalance processes. Besides, the superconducting clusters situated at the contact area periphery may result such the horns [11]. In some measurements the spectra occur not symmetrical, as it is shown in Figs. 1 and 2. The reason for this phenomenon does not consider in present publication.

Because of the unusual type of spectra measured, the standard BTK [9] theory for the description of the current-voltage characteristics can not be used for finding the gap values. For a qualitative estimation the order parameter $\Delta$ and its temperature and magnetic dependences we define it as a half-width of the zero bias minimum at a half-depth of the minimum. Apparently, the depth of this minimum 


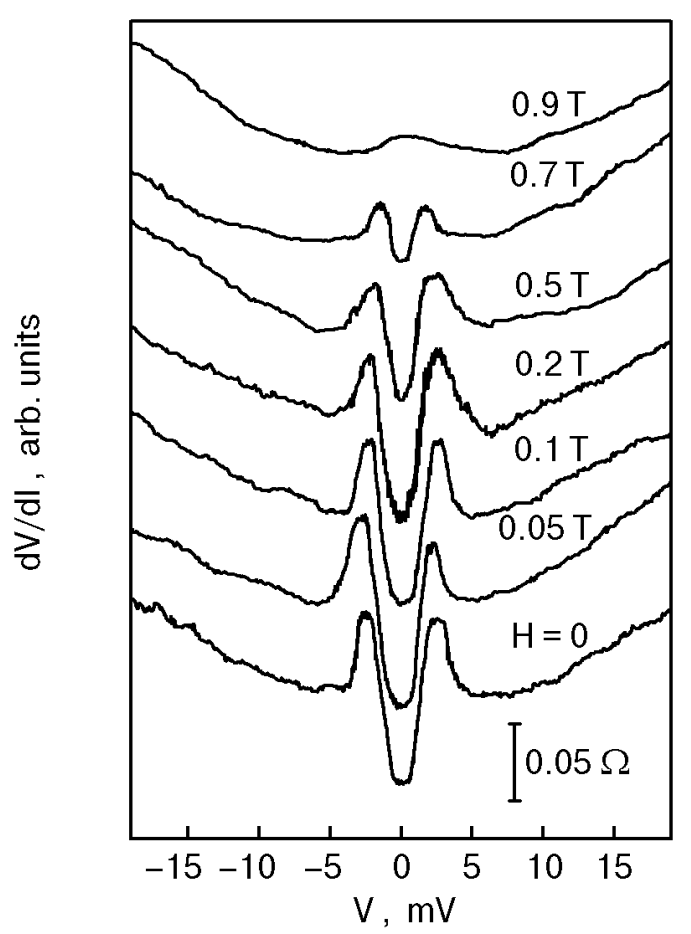

Fig. 2. $d V / d I(V)$ spectra at the different indicated magnetic fields for a point contact between an Ag needle and $\mathrm{YFe}_{4} \mathrm{Al}_{8}$ single crystal with normal contact resistance $R_{n}=5 \Omega$ and critical temperature $T_{c}=6.15 \mathrm{~K}$. Temperature $T=4.2 \mathrm{~K}$. For clarity the curves are shifted vertically. The zero-bias structure disappears at a critical magnetic field $H_{c 2}=0.87 \mathrm{~T}$.

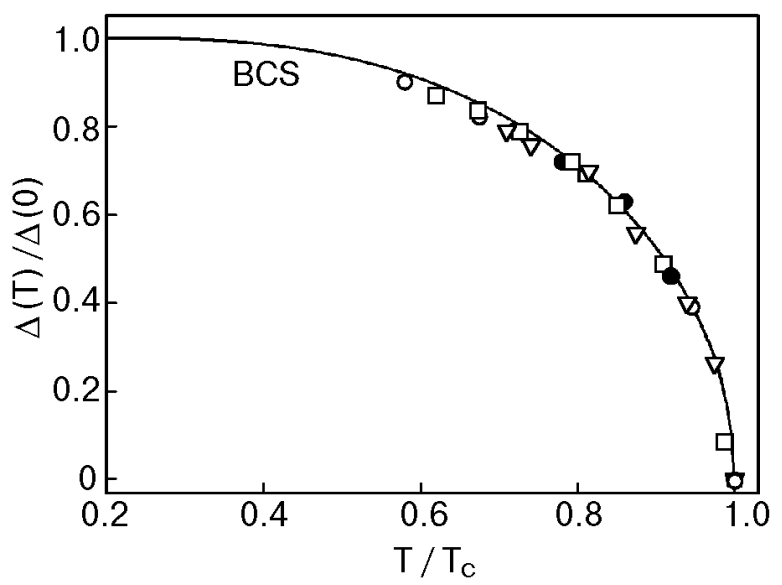

Fig. 3. The order parameter temperature dependences $\Delta(T)$ at zero magnetic field $H=0$ for four series of $\mathrm{Ag}-\mathrm{YFe}_{4} \mathrm{Al}_{8}$ point contact measurements on different areas of $\mathrm{YFe}_{4} \mathrm{Al}_{8}$ single crystal surface. The corresponding critical temperatures $T_{c}$ for different series are: $5.85 \mathrm{~K}$ $(\bullet), 6.15 \mathrm{~K}(\Delta), 6.84 \mathrm{~K}(\square)$, and $7.40 \mathrm{~K}(\bigcirc)$. Related $2 \Delta(0) / k T_{c}$ ratios are: $3.5(\bullet), 4.0(\Delta), 7.2(\square)$, and 5.7 (O). The corresponding critical magnetic fields $H_{c 2}$ are: $5 \mathrm{~T}(\bullet), 0.87 \mathrm{~T}(\Delta), 0.45 \mathrm{~T}(\square)$, and $0.4 \mathrm{~T}(\bigcirc)$. Solid line shows BCS theoretical prediction. also correlates with the order parameter and reflects its temperature and magnetic field dependences. Therefore, we also estimated the $R^{0}=R_{n}-R_{s}$ value, where $R_{n}$ and $R_{s}$ are the resistances at $V=0$ in the normal and superconducting states, respectively. Obtained in such a manner temperature dependences $\Delta(T)$ for four series of PC spectra measured coincide well with BCS theoretical curve as it is seen from Fig. 3. The corresponding $T_{c}$ are $5.85,6.15,6.84$, and $7.40 \mathrm{~K}$. Related $2 \Delta(0) / k T_{c}$ ratios are $3.5,4.0,7.2$, and 5.7 , respectively.

In Fig. 2 one can see that both the width and depth of the minimum in the differential contact resistance reduce when the applied magnetic field increases and gradually disappear upon approaching about $0.9 \mathrm{~T}$. In contrast to the temperature dependences, the magnetic field dependences for all spectra measured do not follow any theoretical predictions. We have met two typical situations which are shown in Fig. 4. It is clearly seen that at the field values higher than $H / H_{c 2} \sim 0.5$, the experimental curves practically coincide. But at lower fields they are different. The curve, represented by open triangles, demonstrates noticeable (about 20\%) enhancement of superconductivity by a weak magnetic field. At higher fields both curves deviate from the pair-breaking theoretical prediction [12] and indicate much more stronger pair-breaking influence of the field. Solid triangular points reflect the $R^{0}(H)$ dependence and correlate fairly with the open triangles, reflecting the $\Delta(H)$ dependence. Both types of

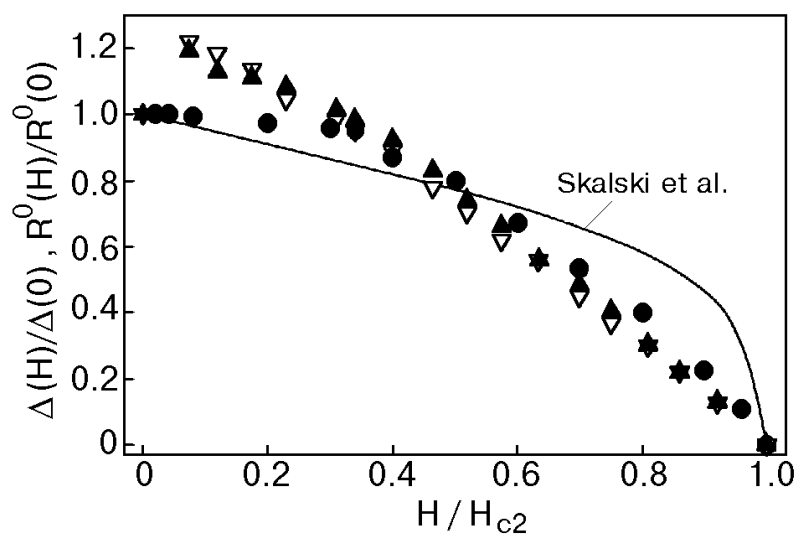

Fig. 4. The order parameter magnetic dependences $\Delta(H)$ at temperature $T=4.2 \mathrm{~K}$ for two series of $\mathrm{Ag}-\mathrm{YFe}_{4} \mathrm{Al}_{8}$ point-contact measurements. The corresponding parameters $T_{c}, H_{c 2}$, and $2 \Delta(0) / k T_{c}$ ratios for data marked by solid circles and open triangles are the same as in Fig. 3. The solid triangles show the magnetic field dependence of the depth minimum $R^{0}=R_{n}-R_{s}$ and correlate well with the open triangles, reflecting the $\Delta(H)$ estimation for the same run of measurements. Solid line shows the pair-breaking theory prediction of Skalski et al. 
data, obtained in the same experiment, unambiguously confirm the occurrence of the superconductivity enhancement effect. Earlier, enhancement of superconductivity in magnetic field was detected in $\mathrm{HoNi}_{2} \mathrm{~B}_{2} \mathrm{C}$ magnetic superconductor [13] and in high- $T_{c}$ materials with magnetic impurities [14]. Probably, all these results are attributed to the reduction of the spin-disorder scattering due to the spin alignment improvement in an applied weak magnetic field as it was discussed in [13]. Moreover, existence of the negative magnetoresistance in $\mathrm{ReM}_{4} \mathrm{Al}_{8}$ compounds in a weak magnetic fields at temperatures just before the superconducting transition $[7,8]$ supports this suggestion.

As was found, the superconductivity in $\mathrm{YFe}_{4} \mathrm{Al}_{8}$ can survive in enough high magnetic fields. The range of $H_{c 2}$ values measured at $T=4.2 \mathrm{~K}$ occurred within $0.4-5 \mathrm{~T}$. The highest value $H_{c 2}=5 \mathrm{~T}$ measured at temperature $T=4.2 \mathrm{~K}$ for sample with $T_{c}=5.85 \mathrm{~K}$ results the coherence length about $80 \mathrm{~A}$. We suppose that significant diversity in $T_{c}$, $H_{c 2}$ and $\Delta$ values, obtained in our experiments, is caused by varying the magnetic states over a sample volume. In other words, magnetic inhomogeneities are inherent for such materials even in single-crystal state.

In summary, we represent the first direct evidence for the existence of superconductivity in the magnetic compound $\mathrm{YFe}_{4} \mathrm{Al}_{8}$ via measurements of Andreev-reflection in the point contacts of $N-S$ type. The anomalous shape of the Andreev-reflection spectra could point to the unconventional character of superconductivity in this compound or magnetic pair-breaking resulting the quasiparticle states within the energy gap.
1. M. B. Maple, in: Advances in Superconductivity, B. Deaver and J. Ruvalds (eds.), Plenum Press, New York (1983).

2. R. J. Cava, H. W. Zandbergen, B. Batlogg, H. Eisaki, H. Tagaki, J. J. Krajewski, W. F. Peck, Jr., E. M. Gyorgy, and S. Uchida, Nature 372, 245 (1994).

3. W. A. Fertig, D. C. Johnston, L. E. DeLong, R. W. McCallum, M. B. Maple, and B. T. Matthias, Phys. Rev. Lett. 38, 987 (1977).

4. P. Schobinger-Papamantellos, K. H. J. Buschow, and C. Ritter, J. Magn. Magn. Mater. 186, 21 (1998).

5. J. A. Paixão, S. Langridge, S. Aa. Sørensen, B. Lebech, A. P. Gonçalves, G. H. Lander, P. J. Brown, P. Burlet, and E. Talik, Physica B234-236, 614 (1997).

6. I. Felner and I. Nowik, J. Magn. Magn. Mater. 54-57, 163 (1986).

7. A. M. Gurevich, V. M. Dmitriev, V. N. Eropkin, L. A. Ishchenko, N. N. Prentslau, and L. V. Shlyk, Fiz. Nizk. Temp. 25, 15 (1999) [Low Temp. Phys. 25, 10 (1999)].

8. A. M. Gurevich, V. M. Dmitriev, V. N. Eropkin, B. Yu. Kotur, N. N. Prentslau, W. Suski, A. V. Terekhov, and L. V. Shlyk, Fiz. Nizk. Temp. 27, 1308 (2001) [Low Temp. Phys. 27, 967 (2001)].

9. G. E. Blonder, M. Tinkham, and T. M. Klapwijk, Phys. Rev. B25, 4515 (1982).

10. Y. de Wilde, J. Heil, A. G. M. Jansen, P. Wyder, R. Deltour, W. Assmus, A. Menovsky, W. Sun, and L. Taillefer, Phys. Rev. Lett. 72, 2278 (1994).

11. O. I. Shklyarevskii, A. M. Duif, A. G. M. Jansen, and P. Wyder, Phys. Rev. B34, 1956 (1986).

12. S. Skalski, O. Betbeder-Matibet, and P. R. Weiss, Phys. Rev. 136, A1500 (1964).

13. L. F. Rybaltchenko, A. G. M. Jansen, P. Wyder, L. V. Tjutrina, P. C. Canfield, C. V. Tomy, and D. McK. Paul, Physica C319, 189 (1999).

14. V. M. Dmitriev, L. A. Ishchenko, and N. N. Prentslau, Fiz. Nizk. Temp. 24, 624 (1998) [Low. Temp. Phys. 24, 471 (1998)]. 